\title{
Assessment of Exposure to Magnetic Fields in Occupational Settings
}

\author{
Hirofumi SaKurazawa ${ }^{1}$, Akio Iwasaki ${ }^{2}$, Toshiaki Higashi ${ }^{2}$, Takeo NAKAYAma ${ }^{3}$ and \\ Yukinori KUSAKA ${ }^{1}$
}

${ }^{1}$ Department of Environmental Health, School of Medicine, Fukui Medical University, ${ }^{2}$ Department of Work Systems and Health, Institute of Industrial Ecological Sciences, University of Occupational and Environmental Health and ${ }^{3}$ Department of Medical System Informatics, Kyoto University Graduate School of Medicine, Japan

\begin{abstract}
Assessment of Exposure to Magnetic Fields in Occupational Settings: Hirofumi Sakurazawa, et al. Department of Environmental Health, School of Medicine, Fukui Medical University-Objective-It is important to have data about occupational magnetic field intensity to consider the contribution of occupational magnetic field exposure on the human body. We conducted research on exposure to occupational magnetic fields and tried to qualify data on the distribution of magnetic field' intensity in certain general working environments with individual measurements. Subjects and Methods-We performed sample research on the exposure to lowfrequency magnetic fields of workers in certain occupations and in the working environment. We also assessed the relationship between working environmental magnetic field distribution and individual exposure. Results-Some occupations were found to be exposed to high magnetic fields. We observed that some workspaces, such as the transformer substation, generally had a uniform and high magnetic field measurement but employees were exposed to a lower intensity. We also found that welders were exposed to high magnetic fields at about $600 \mu \mathrm{T}$ in a very short time but with a geometrical value of $0.08 \mu \mathrm{T}$. Conclusion-The determination of administrative levels and control levels, not only of the time weighted average of threshold limits or short term exposure limits, but also ceiling limits should be considered. More systematic research is necessary to determine variables such as operating conditions, measuring position, and frequency bands. Also, further studies will be needed to make a job-exposure matrix for the magnetic fields for each occupation type and to combine it with exposure in non-occupational settings
\end{abstract}

Received Nov 5, 2002; Accepted Jan 9, 2003

Correspondence to: H. Sakurazawa, Department of Environmental Health School of Medicine Fukui Medical University Matsuokacho Fukui 910-1193, Japan such as commuting and ordinary life situations to explore the causal relationship between exposure to magnetic fields and disease.

(J Occup Health 2003; 45: 104-110)

Key words: Electromagnetic fields, Occupational exposure, Working environment, Exposure measurement

Since Wertheimer-Leeper's report in 1979'1), various reports on the influence of electromagnetic fields on human health have been published. Most of them have been concerned with an increase in the risk of malignant diseases e.g. leukemia, brain tumors.

In recent years, several reports of epidemiological research concerning the relationship between electromagnetic fields and malignant diseases have been published. Some of these original papers or reviews showed that magnetic fields increased the risk of cancer but some did not. There is no obvious explanation for the discrepancies among the major studies. These could be due to real differences among populations or methodological errors in individual studies.

Exposure measurements from electromagnetic fields representing each occupational category differ markedly across workplaces and industries ${ }^{2-11)}$. In these studies, measurements for a given occupation were generally made at one worksite. The assumption of equivalent magnetic field exposure across settings is tenuous, particularly comparing diverse workplaces ${ }^{2)}$ within the electric utility environment ${ }^{4,5}$. Identical average intensity or cumulative exposure may mask important differences in the temporal patterns of exposure.

Furthermore, the approach to grouping workers, selecting individuals for exposure measurement, the types of instruments used, and use of the data to classify and analyze exposure (cumulative time-weighted average (TWA) or multiple indices) may all contribute to divergent 
results.

Delpizzo ${ }^{6)}$ noted that there was substantial misclassification of individual exposures based on using job titles. In the 1990s, investigators had begun to incorporate magnetic field measurements into the construction of job-exposure matrices as a method of estimating the electromagnetic field exposure level. This is done by measuring the standard exposure level of each job type and adding an estimated exposure value based on the work type and length of employment. Nevertheless, the risks from electromagnetic field exposure have sometimes weakened according to the results of these studies ${ }^{7,8)}$. The exposure misclassification resulting from mis-judgment is virtually certain to be no different. As a result, the precision of the exposure estimates will be limited, resulting in the "classical" type of misclassification and attenuation of the dose-response relationship. These exposure assessments are also limited because they use information based only on occupation and the time of diagnosis.

Another problem is the assessment of historical exposure to magnetic fields. Some recent studies have documented an individual's time at a particular job during the past several years with an additional measurement of the present exposure to magnetic fields ${ }^{9}$. This allows a job-exposure matrix to be made to get a more accurate assessment of historical exposure to a magnetic field in the workplace. Therefore, the current reality is that we need further research in this area.

In Japan, there has been little epidemiological research based on measured values of exposure to magnetic fields. Japan differs from Western countries in voltage and peripheral elements including electric wires, position of power cables and operating procedures. Information on exposure levels to magnetic fields in occupational settings is important for evaluation of the dose-response relationship in future epidemiological studies and working environment control, but Japan's accumulation of data on exposure to magnetic fields in public places and in occupational settings is smaller than in other countries. A first step in a complete surveillance effort is to prioritize industries in order of potential magnetic field exposures for subsequent personal monitoring and source characterization. In this study, we selected occupational settings where the workplace exposure to magnetic fields could be considered relatively high, and we used individual exposure-measuring instruments to collect sample values. We also performed working environment measurement. We imitated the "sampling points $A$ " method and the "sampling point B" method which have been used in order to assure accurate working environment measurement and established control levels for the working environment in the Working Environment Measurement Law in 1975 and the Working Environment Measurement Standards (Ministry of Labour Notification
N0. $46,1976^{12)}$.

\section{Subjects and Methods}

\section{Sampling Strategy}

Measurements of magnetic fields in a work environment were first conducted in a transformer substation (A) of an electric power company as a one unit work environment, which could be considered to be a work site with relatively high exposure to magnetic fields. We selected this work environment to clarify whether there is no remarkable variation in exposure or consistency in individuals even when workers' positions vary in this set unit. We imitated the "sampling points $A$ " method. We drew a line lengthwise every $0.5 \mathrm{~m}$ from the entrance, then sideways and made a grid with the intention of choosing sampling points at random. Nevertheless, machinery prevented us from measuring in this fashion so we measured the magnetic field at 3 points on every lateral line, "50 cm from switch gear", "50 cm from transformer" and "around the distributing board". We also measured where the worker was considered to be at maximum exposure, the "sampling points B" method. We also measured personal exposure.

Measurements were next conducted in an ordinary office (B), with relatively low exposure to magnetic fields. We measured magnetic intensity and electric intensity around office machinery.

We also measured other occupational workplaces where permitted by informed consent with a privacy policy, conducting personal monitoring and the "sampling points B" method in order to test whether occupational types are a surrogate for magnetic field exposures. Personal monitoring surveys were conducted by 8 -h timeweighted average (TWA) ${ }^{13}$. The time weighted average of an eight-hour workday is calculated with the following equation

$$
V_{(T W A)}=\frac{V a T a+V h T h+\ldots+V i T i+\ldots}{8}
$$

where $V i$ is a value within a certain time during a period $T i$ when the value is constant.

In the office environment, the workers were exposed to the magnetic fields during all working hours. Using a TWA of the duration of exposure, their cumulative exposure value may be high when compared with the residential exposure level. Full work shifts spent close to low current sources may produce greater exposure than occupations spending short work periods close to high current sources and the remainder of the working day in low exposure situations. Thus we measured the exposure values for each job description in the electric power company. 


\section{Monitoring Strategy}

In order to measure the exposure value, we used the EFA-3 (manufactured by Wandel \& Goltermann, Inc.). The frequency band of the EFA-3 is $5-30 \mathrm{kHz}$ the measuring range of the electric field is $0.1 \mathrm{~V} / \mathrm{m}-100 \mathrm{kV} /$ $\mathrm{m}$, and the magnetic field is $10 \mathrm{nT}-10 \mathrm{mT}$ ). It was used by a Class 1 Working Environment Measurement Expert stipulated in Article 2 of the Working Environment Measurement Law (Law No. 28 of May 1, 1975) during the survey at each facility. The EFA-3 contains a frequency counter, real triaxial (isotropic) probes and electronic signal processing that measures and analyze the field components in the three spatial axes $\mathrm{x}, \mathrm{y}$ and $\mathrm{z}$. It measures not only peak value but also the three rootmean-square (RMS) components which yields the RMS vector magnitude of the extremely low frequency (ELF) magnetic flux density.

In order to measure individuals magnetic field exposure, we used a memory-type portable exposuremeasuring instrument, the ML-1 (Manufactured by Enviro Mentor, Inc.), in addition to the EFA-3. The frequency band of the ML-1 is $30-2,000 \mathrm{~Hz}$ and the measuring range of the magnetic field is $0.05 \mu \mathrm{T}-100$ $\mu \mathrm{T}$. The ML- 1 contains three orthogonal induction coils and electronic signal processing that measures and registers the RMS value of the magnetic alternating fields in the $\mathrm{x}, \mathrm{y}$ and $\mathrm{z}$ directions, irrespective of the direction in which the instrument is pointing in relation to the magnetic field. The ML-1 can record and store a series of measurements at intervals of between 1 and $150 \mathrm{~s}$, and can store up to 8,192 readings. It measures the three RMS components, yielding the RMS vector magnitude of the extremely low frequency (ELF) magnetic flux density in microtesla $(\mu \mathrm{T})$.

The measurements were conducted in an indoor transformer substation (A), office (B) and other places, which, because of the privacy policy, we could not describe in detail. A Class 1 Working Environment Measurement Expert, accompanied us and gave us some suggestions. We measured the indoor transformer (A) at every point placed by the EMF-3 probe mounted on a one-meter tall stand. We also measured where every employee in the transformer substation (A) was considered to be at risk of maximum exposure. We also measured personal monitoring by the ML- 1 attached to the waist of the worker.

The Class 1 Working Environment Measurement Expert walked through ${ }^{14)}$ the office (B) and searched where to take measurements at work stations where workers spent long periods of time near magnetic field sources. We placed the probe mounted on a one-meter tall stand as close as possible (about $30 \mathrm{~cm}$ ) to an individual's work station. We also measured the amount of exposure to the electric field during work time.

The individual measurements were conducted on VDT operators and 8 office workers from (A) and (B) and 2 thermo-electric plant operators from (A). Furthermore, 3 electric furnace operators, 4 gas shield door arc welders, 5 train crew members, 2 railroad maintenance workers, and 2 operators were also measured in different industries. The sample measurements were taken every $5 \mathrm{~s}$ for a full working day on each worker, but in the case of the thermoelectric power plant workers, it was taken every 10 or $20 \mathrm{~s}$ for a working day because of their shift work. The ML-1 was attached to the waist of the worker, and environment measurement to detect peak magnetic field exposure was taken by the Class 1 Working Environment Measurement Expert with the EFA-3 set on the floor at a distance of 1 meter from the subject. After each measurement, data were downloaded to a portable computer. Explanatory variable information which may influence exposure was recorded by observing the activities of the workers. All magnetic field strengths were measured at $60 \mathrm{~Hz}$.

The manufacturer detected no significant change in calibration after the course of the study.

\section{Results}

The results of the utilization of the "sampling points $A$ " method survey in workplace measurements in the indoor transformer substation (A) are shown in Table 1. The table has a lengthwise line number every $0.5 \mathrm{~m}$ from the entrance door, and each 3 measured point magnetic fields including " $50 \mathrm{~cm}$ from switch gear", "50 cm from transformer" and "around the distributing board" along the each line. In this working environment, relatively high exposure values to magnetic fields from 1.0 to 7.0 $\mu \mathrm{T}$ were measured in spite of the variation in measuring periods. In other words, workers were exposed to chronically high magnetic fields. The factor having the most effect on an individual's cumulative personal exposure is thought to be the amount of time spent in a particular position with high exposure near the source. The exposure value for each job description in the transformer substation (A) and the office (B) is shown in Table 2. Personal exposure and the explanatory variables were measured for a full working day in each worker. Considering one engineer's exposure, the $8 \mathrm{~h}$ TWA value ranged from $0.5 \mu \mathrm{T}$ to $3.0 \mu \mathrm{T}$ and peak exposure values differed greatly among job descriptions, because distances from the magnetic field source, working conditions and time varied in each case.

On the other hand, in an ordinary office where OA machines were in use, relatively low magnetic field exposure values from 0.4 to $1.88 \mu \mathrm{T}$ were measured around the OA machines (Table 3 ). According to Table 2 , despite the limited sample number, the office worker's cumulative exposure value may be low.

Based on the above results, we recorded the situation of each work site's exposure to magnetic fields in Table 
Table1. Transformer Substation Magnetic Field Measurements

\begin{tabular}{ccccc}
\hline $\begin{array}{l}\text { Lengthwise } \\
\text { Line No. }\end{array}$ & $\begin{array}{l}\text { Distance from } \\
\text { the entrance } \\
\text { door }(\mathrm{m})\end{array}$ & $\begin{array}{l}50 \mathrm{~cm} \text { from } \\
\text { Switch Gear } \\
(\mu \mathrm{T})^{\# 1}\end{array}$ & $\begin{array}{l}50 \mathrm{~cm} \text { from } \\
\text { Transformer } \\
(\mu \mathrm{T})^{\# 2}\end{array}$ & $\begin{array}{l}\text { Around Distributing } \\
\text { Board }(\mu \mathrm{T})^{\# 3}\end{array}$ \\
\hline 1 & 0.5 & 3.5 & $3.4^{*}$ & 1.9 \\
2 & 1.0 & 4.0 & $3.8^{*}$ & 1.1 \\
3 & 1.5 & 4.0 & $4.2^{*}$ & 6.0 \\
4 & 2.0 & 6.0 & $6.5^{* *}$ & 2.5 \\
5 & 2.5 & 7.0 & $5.5^{* *}$ & 1.0 \\
6 & 3.0 & 5.5 & $7.0^{* *}$ & $-{ }^{\# 4}$ \\
\hline
\end{tabular}

*: 60,000V Side, **: 6,000V Side

\#1: Time weighted average of values in $10 \mathrm{~min}$, \#2: Time weighted average of values in 20 to $30 \mathrm{~min}$ \#3: Time weighted average of values in 3 to $4 \mathrm{~min}$, \#4: Obstacles preventing us from measuring

Table 2. Summary of Exposure Value of Personal Exposure Measurements

\begin{tabular}{llcc}
\hline Employee & Occupational Type & $\begin{array}{l}\text { Estimated } \\
8 \mathrm{~h} \text { TWA }(\mu \mathrm{T})\end{array}$ & Maximum Value $(\mu \mathrm{T})$ \\
\hline Engineer $A$ & Operator on Duty & 0.5 & 263.5 \\
Engineer $B$ & Maintenance & 1.0 & 74 \\
Engineer $C$ & Operator & 3.0 & 60 \\
Engineer D & Maintenance & 1.0 & 40 \\
Office Worker $E$ & Desk Work & Less Than 0.1 & 1.88 \\
\hline
\end{tabular}

Table 3. Office Electromagnetic Field (Distance: $30 \mathrm{~cm}$ )

\begin{tabular}{lcc}
\hline Measured Machinery & Electric Intensity $(\mathrm{V} / \mathrm{m})$ & Magnetic Intensity $(\mu \mathrm{T})$ \\
\hline LAN Terminal & 2.6 & 0.40 \\
Old Type Desk-top Personal Computer & 4.2 & 1.68 \\
Desk-top Personal Computer & 2.3 & 0.80 \\
Old Type Word Processor & 8.6 & 1.88 \\
Residential TV & 25 & 1.88 \\
\hline
\end{tabular}

Time weighted average of values in $3 \mathrm{~min}$.

Table 4. Occupational Type and Exposure to Low Frequency Electromagnetic Field

\begin{tabular}{|c|c|c|c|c|c|c|}
\hline Occupation Type & $\mathrm{n}$ & $\begin{array}{l}\text { Mathematical } \\
\text { Mean Value }\end{array}$ & $5 \%$ Value & $95 \%$ Value & $\begin{array}{l}\text { Geometrical } \\
\text { Mean Value }\end{array}$ & Maximum Value \\
\hline VDT Operator & 8 & 0.17 & 0.06 & 0.34 & 0.13 & 0.89 \\
\hline $\begin{array}{l}\text { Thermoelectric } \\
\text { Plant Operator }\end{array}$ & 2 & 0.49 & 0.42 & 0.47 & 0.43 & 263.5 \\
\hline Train Crew & 5 & 0.56 & 0.07 & 2.97 & 0.25 & 7.91 \\
\hline $\begin{array}{l}\text { Rail Road } \\
\text { Maintenance } \\
\text { Worker }\end{array}$ & 2 & 0.61 & 0.02 & 0.12 & 0.18 & 16.7 \\
\hline Welder & 4 & 0.78 & 0.02 & 7.51 & 0.08 & about 600 \\
\hline $\begin{array}{l}\text { Electric Furnace } \\
\text { Operator }\end{array}$ & 3 & 9.91 & 0.01 & 70.7 & 0.19 & 35.7 \\
\hline
\end{tabular}


4. Four exposure metrics were obtained from the ML-1: mathematic mean, $5 \%$ value, $95 \%$ value and geometric mean. The maximum value was obtained by the EFA-3. Electric power plant operators' geometric mean exposure was $0.43 \mu \mathrm{T}$, about 5 times higher than that of welders. The railroad maintenance operator exposure average was $0.18 \mu \mathrm{T}$, and of the train crew was $0.25 \mu \mathrm{T}$ and higher than that of the welders. Electric furnace operators had the risk of exposure to very high current sources. We also found when using the EFA-3 that welders were exposed to a high magnetic field, of about $600 \mu \mathrm{T}$. High temperature welding could influence the readings on the EFA-3 and prevent us from finding the exact maximum value. From the measurement of welders with the ML1 , the geometrical value was only $0.08 \mu \mathrm{T}$, indicating a significant difference between the maximum value and the geometrical value. Of course, the ML-1 measuring range for the magnetic field is $0.05 \mu \mathrm{T}-100 \mu \mathrm{T}$ and cannot detect higher values.

\section{Discussion}

Industrialization has introduced many electric machines to assembly lines and offices. It has been reported that exposure to electromagnetic fields around high voltage cables or electric construction increases the incidence of leukemia ${ }^{2-4)}$, brain tumors ${ }^{3)}$ and breast cancer $^{15)}$. As carcinogenicity is based on epidemiological associations, a recent US government review classified extremely low frequency $(E L F=3-3000 \mathrm{~Hz})$ magnetic fields as a "possible carcinogen"16). On the other hand, in vivo studies have found almost no increase in either leukemia or brain cancer from magnetic field exposure, although such animal experiments have limitations as predictors of human carcinogenesis with environmental $\mathrm{EMF}^{17)}$. There have been some reports suggesting that evidence of a relationship between carcinogenicity and exposure to electromagnetic fields from electric machines is minimal ${ }^{15}$. There is no clear evidence of a dose-response relationship with workers in jobs with higher exposure to magnetic fields more likely to suffer from malignant carcinoma than in jobs with lower exposures. These could be caused by the difficulty of evaluating the effects of magnetic fields on the human body. For example, in evaluating exposure to magnetic fields, a method taking into account past and total cumulative exposure has been difficult to develop and easily causes measurement bias. In terms of comparative studies or retrospective cohort studies, it is difficult to directly measure the amount of past cumulative exposure to magnetic fields. In residential settings, instead of measuring residential cumulative exposure to magnetic fields directly, a method called the "Wire Code Method")" is used as a surrogate. By this method, researchers estimate exposure values by the distribution of the electric power volume or cables, and the cumulative or past exposure value is calculated ${ }^{11,18)}$.
Savits") concluded that estimates based on the "Wire Code method" could be an indication of the exposure value inside the house. In order to evaluate the relationship between the exposure level and health effects, Savits reported that instead of using the cumulative exposure value, the appropriate exposure value could be estimated by applying a method called job-exposure matrices to the electromagnetic field ${ }^{8)}$. In his report, he applied jobexposure matrices to the electromagnetic field as a method of estimating the electromagnetic field exposure level by measuring the standard exposure level of each job type and adding an estimated exposure value based on the work type and years of employment. The current study was designed to attempt to make more accurate assessments of electromagnetic field exposure. Considering this situation, explanatory variables should be investigated.

The results of this study provide new information on ambient magnetic fields magnitudes in diverse occupational settings. The utilization of the "sampling points A" method and "sampling points B" method survey provided a systematic method to rank industrial magnetic field environments, which permitted surveys to be conducted in a wide variety of industries/facilities where magnetic field levels have not been previously documented.

The survey also identified some high magnetic field sources, such as an electric furnaces and welding.

The survey collected important qualitative information about sources that could help to explain magnetic field measurements. In the transformer substation, there were some areas of high intensity magnetic fields (Table1), but this conclusion is limited because the duration of measurement was short. Although the raw data in Table 2 seemed to show the correlation between electric intensity and magnetic intensity, the magnetic field intensity due to office machines was low. The reliability of this conclusion is limited by the non-random selection of facilities (Table3). From Table 3, it is seen that the desk worker spent less time in areas with strong fields. We have also noted that engineers seldom worked alongside equipment with high magnetic fields, for example, a power panel. Tables 1-3 show that many moderate magnetic field sources have created high ambient fields and a greater potential for worker exposure. In addition, this survey collected information about the number of workers moving from area to area who do not regularly occupy a fixed workstation. We therefore measured them by personal monitoring and used the analysis of the magnetic field surrogates since the ML-1 is likely to indicate overall worker exposure in a facility. The measurement technique is limited by the specifications of the ML-1. Some mathematical mean values were 10 times larger than the geometrical mean values. The ML-1 has a band width of $30-2,000 \mathrm{~Hz}$ and a dynamic range of $0.05 \mu \mathrm{T}-100 \mu \mathrm{T}$ and will not accurately record the magnitude of fields outside this 
range because some data might be outside the range. Furthermore, the ML-1 meter only measures the RMS vector magnitude of the extremely low frequency magnetic field and not other field characteristics, potentially important to biology ${ }^{19}$. It is also thought that welders used electricity momentarily or welded for a short period, and for the remainder of the working day they worked in low exposure situations. In order to characterize harmonic frequencies, high frequency transients, and equipment operating at frequencies outside the range of the ML-1 instrument, a more detailed survey is needed with a waveform-capturing instrument ${ }^{20)}$. In this research, the mathematical mean was found by taking the total of each worker's measured mathematical mean value and dividing this total by the number of workers. The geometrical mean value was determined by taking the total workers' geometrical means and dividing it by the number of workers. The geometrical means may be affected by the number of sources as well as the source magnitudes ${ }^{14}$. A high geometric mean could indicate that the worker was exposed to a relatively high magnetic field for a short time but the poor correlation can be explained by the relationship between the electric power and magnetic fields, which is strongly modified by the source voltage and wiring configuration ${ }^{14)}$. In order to make things clear, it is necessary to take a measurement in the occupational setting where the workers are constantly exposed to a relatively high magnetic field and record the distribution of the magnetic field with one meter equipped for data collection and having a wide range. To determine the matrices of job-exposure to the magnetic fields in various occupation types, it will be necessary to conduct many preliminary direct surveys on exposure to magnetic fields in various occupational settings.

The field strength was influenced by such conditions as the distance from the generating source, usage, working conditions and duration of work time. Given these limitations, magnetic field surveillance data cannot be used at the present time to guide exposure control efforts, the traditional role of hazard surveillance. But from the surveillance data, it can identify magnetic field sources and probably provide guidance for the personal monitoring and analysis of future epidemiological studies. The results of this report can assist the description of magnetic field exposure because the characteristics of the measurements of magnetic fields in work environments had considerable variation, so that a combination of measurement strategies should be considered. We also clarified that measurements need to be conducted not only in field surveys but also in personal monitoring surveys. The advantage of the "sampling points A" method and "sampling points B" method survey is that workers may not influence the data collection because we place the monitor close to the electrical equipment. In addition, the survey was easily conducted and workers did not appear to be disturbed during the production processes. Thus the combination of surveys can allow calculation of an exposure estimation formula considering variables in operating conditions, measurement position, and frequency band in actual situations.

Our research also indicated that the distribution of exposure differed depending on the job type. This point should be taken into consideration when evaluating exposure to magnetic fields in epidemiological research. It should also be recognized that the measured value is not always the typical general exposure value for the occupation type observed.

An important goal is to assess the relationship between the "sampling points A" method and "sampling points $B$ " method survey and personal measurements. Determination of administrative levels and control levels, with not only the time weighted average of the threshold limit value or short term exposure limits, but also the ceiling limits should be considered.

It will also be necessary to make an exposure index by combining the measured results, because the value of each magnetic field is not constant or typical even in similar occupational fields. Results from the "sampling points A" method and "sampling points B" method survey cannot be compared directly with the TWA of magnetic field magnitudes measured by personal monitoring because workers may move in and out of the magnetic fields.

In the design of future research, scientists should concentrate on improving the exposure assessment and accounting for various confounders. The study on occupational exposure should include environmental and/ or residential exposure, in addition to the information from the occupation and the result of direct or indirect measurement of the electromagnetic field in the workplace. This is most easily accomplished with the use of a function-equipped portable monitor attached to the subject as done in this study, in conjunction with an activity diary or an estimate of time spent in different activities. Finally, data on other risk factors should also be gathered, including exposure to carcinogenic chemicals and viruses.

\section{Conclusion}

In order to assess the exposure to magnetic fields in an occupational setting, we conducted sample measurements of exposure to extra low frequency electromagnetic fields. Our results showed that employees of electric companies or railroad maintenance workers were exposed to the same intensity level of electromagnetic fields as welders or inductive heating apparatus operators. Also, other factors including distance from the generating source, usage, operating conditions and duration of working time influenced the electromagnetic field exposure. This 
research was conducted in specific and limited situations, so that more systematic research is needed to allow calculation of an exposure estimation formula considering variables such as operating conditions, measurement position, and frequency band, as well as actual exposure data in different jobs.

Data accumulation is also necessary to determine a matrix of job-exposure to the electromagnetic field for each occupation type and to combine it with exposure from non-occupational settings to explore the relationship between exposure to electromagnetic fields and diseases.

Acknowledgments: This study was supported in part by a Grant-in-Aid for Cancer Research (10S-1) from the Japanese Ministry of Health and Welfare, 1997-1999

\section{References}

1) $\mathrm{N}$ Wertheimer and E Leeper: Electrical wiring configrations and childhood cancer. Am J Epidemiol 109, 273-284 (1979)

2) B Flodeus, T Persson, C Stenlund, A Wennberg, A Ost and B Knave: Occupatinal exposure to electromagnetic fields in relation to leukemia and brain tumors: a casecontrol study in Sweden. Cancer Causes Control 4, 465-476 (1993)

3) JD Sahl, MA Kelsh and S Greenland: Cohort and nested case-control studies of hematopoietic cancers and brain cancer among electric utility workers. Epidemiol 4, 1104-1114 (1993)

4) G Thériault, M Goldberg, AB Miller, B Armstrong, $P$ Guennel, J Deadman, E Imbernon, T To, A Chevalier, D Cyr and C Wall: Cancer risks associated with occupational exposure to magnetic fields among electric utility workers in Ontario and Quebec, Canada, and France: 1970-1989. Am J Ind Med 139, 550-572 (1994)

5) A. Savitz David and P. Loomis Dana: Magnetic field exposure in relation to leukemia and brain cancer mortality among electric utility workers. Am J Epidemiol 141, 123-134 (1995)

6) V Delpizzo: An apparently incongruous exposureresponse relationship resulting from the use of job description to assess magnetic field exposure. Scand J Work Environ Health 18, 242-245 (1992)

7) DA Savits and NE Pearce: Methodological issues in the epidemiology of electromagnetic fields and cancer. Epidemiol Rev 11, 59-78 (1989)

8) DA Savits and NE Pearce: Update of methodological issues in the Epidemiology of electromagnetic fields and cancer. Epidemiol Rev 15, 558-566 (1993)

9) H Wang, Y Murai, S Nomura, M Sekine, S Sokejima, H Sakai and S Kagamimori: A Meta-analysis of epidemiological studies on the relationship between occupational electromagnetic field exposure and the risk of adult leukemia. Environmental Health and Preventive Medicine 5, 43-46 (2000)

10) SJ London and DC Thomas: Exposure to residential electric and magnetic fields and risk of childhood leukemia. Am J of Epidemiol 134, 923-937 (1991)

11) RK Severson and RG Stevens: Acute nonlymphocytic leukemia and residential exposure to power frequency magnetic fields. Am J Epidemiol 128, 10-20 (1988)

12) Working environment measurement system in Japan. Industrial Health Division Industrial Safety and Health Department Labour Standards Bureau Ministry of Labour, eds. Tokyo: Japan Association for Working Environment Measurement, 1986: 30-103.

13) ACGIH. Documentation of the threshold limit values and biological exposure indices, 6th ed. Cincinnati: ACGIH, 1991.

14) MM Methner and JD Bowman: Hazard surveillance for industrial magnetic Fields: I. Walkthrough survey of ambient fields and sources. Ann Occup Hyg 44, 603614 (2000)

15) PF Coogan, RW Clapp, PA Newcomb, TB Wenzl, G Bogdan, R Mittendorf, JA Baron and MP Longnecker: Occupational exposure to 60-hertz magnetic fields and risk of breast cancer in women. Epidemiol 7, 459-464 (1996)

16) National Institute for Environmental Health Sciences, National Institute of Health. NIEHS REPORT on Health Effects from Exposure to Power-line Frequency Electric and Magnetic Fields. Research Triangle Park, NC: National Institute for Environmental Health Sciences, NIH Publication No.99-4493, 1999.

17) EMF RAPID (Electric and Magnetic Fields Research and Public Information Dissemination) Program. EMF Science Review Symposium: Breakout Group Reports for In vivo and Clinical Research Fïndings. Research Triangle Park, NC: National Institute for Environmental health Sciences, 1998.

18) M Feychting and AAhlbom: Magnetic fields and cancer in children residing near Swedish high-voltage power lines. Am J Epidemiol 138, 467-481 (1993)

19) PA Valberg: Designing EMF experiments: What is required to characterize "exposure"? Bioelectromagnetics 16, 396-401 (1995)

20) JD Bowman and MM Methner: Hazard surveillance for industrial magnetic fields: II. Field characteristics from waveform capture. Ann Occup Hygiene 44, 615633 (2000) 